\title{
Study of Retail Applications with Virtual and Augmented Reality Technologies
}

\section{T. Senthil Kumar,}

Associate Professor,

Computer Science and Engineering Department,

Amrita School of Engineering,

Amrita Vishwa Vidyapeetham,

Ettimadai P.O, Coimbatore - 641112,

Tamil Nadu, India.

Email: t_senthilkumar@cb.amrita.edu

Abstract: The rapidly emerging virtual reality (VR) and augmented reality (AR) technologies have greatly improved the digital shopping experience and retail selling environment. In terms of practical applications and academic research, fragmentation in VR and AR contributes to the technology's multidisciplinary roots in terms of applications. In this paper, the retail applications and research works that make use of VR and AR technology are compared and analyzed. The implementation, consumer acceptance, applications, issues and other related terms are compared. This study establishes a foundation for future work in the retail applications field.

Keywords: Virtual Reality, Augmented Reality, Usability engineering, human computer interface, retail industry

\section{Introduction}

Since the early $21^{\text {st }}$ century, retailers have incorporated modern technologies to improve the shopping experience as well as the store environment in both online and physical locations [1]. The digital signage, online product customization and visualization, interactive visualization and other direct customer experiences offered by these technologies in both online and physical store plays a major role in gaining significant customer attraction and also these advanced technologies offer an enhanced shopping experience to the customers 
[2]. The retail environments are increasingly making use of the rapidly evolving virtual reality (VR) and augmented reality (AR) technologies in their applications [3]. The AR technique combines the physical view of the user with the digital information generated by the computer into a single environment. The real and virtual world are blended such that virtual elements, videos, textual data or images may be added with the help of a virtual layer in the physical environment, which is viewed by the user on a real time basis. Mobile phone, webcam or digital camera is used for capturing the real-time data in AR platform [4].

Interaction between the customer and virtual products is enabled by using innovative and creative ways by capturing the attention of customers with the devices like fixed interactive screens, projectors, wearable headsets, tablets or smartphones [5]. Videos, GPS data, sound, media richness and product simulations help in creating an experiential value. The brand image is improved by establishing product visualization and smooth interaction between the consumer and virtual items by using the AR shopping experience, which leads to the purchase of products from the consumers [6]. On the other hand, the sensory experience of the real world is completely blocked out by using headset as a wearable device in the VR technology, wherein an entertaining and virtual 3D world immerse the user in an innovative and engaging shopping experience [7]. The movement of the user's limbs may be captured via motion tracking along with the head movements thereby enabling them to physically move within the virtual world and interact in a real time basis. Credible immersive virtual effects must be conferred along with the comfort of the headwear, which contribute to the successful implementation of VR technology [8]. The virtual world in VR is an interactive computer simulation-based medium that provides the sensation of being psychologically immersed in the simulation environment by augmenting or replacing input to the user's activities through single or multiple senses [9].

Academic interests have increased largely with the rapid advancements in VR and AR technologies, which lead to various applications and developments [10]. In real-time as well as in academic literature, these technologies have been substantially considered despite its origin with very few early exploratory work performed in the initial stages. There is a huge gap in research literature and practical applications of VR and AR in the retail industry context and various other specific applications. The current debates, developments, issues and 
challenges in the retail industry and application of VR and AR technologies are as critical as the interdisciplinary nature of the subject [11]. This largely helps in identification of future research directions in this domain. This review examines the recent advancements of VR and $\mathrm{AR}$ in retail industry and proposes the future research direction to bridge the research gap.

\section{Related Works}

The terms virtual reality, augmented reality and online shopping are compared on Google trends and the trendline is obtained for a duration of five years [12]. From figure 1 it is evident that, during the early 2020 , there has been a sudden rise in the combination of these keywords being searched on Google. It has been sustaining between 75-50 over the last year. Before a decade, little or no material is available for online retailing on the use of VR/AR technology despite the existence of several independent e-commerce websites. Online retailers has not accepted this technology when real-time user based communication and virtual 3D-modeling benefits were proposed by the use of AR/VR technology in e-commerce in early 2000s [13]. Alibaba's Buy+ VR platform, LensKart, VR shopping kiosks, Nykaa, Amazon's AR View and several furnishing retailers have now established a dedicated VR/AR retailing platform online to offer interactive and immersive shopping experience [14].

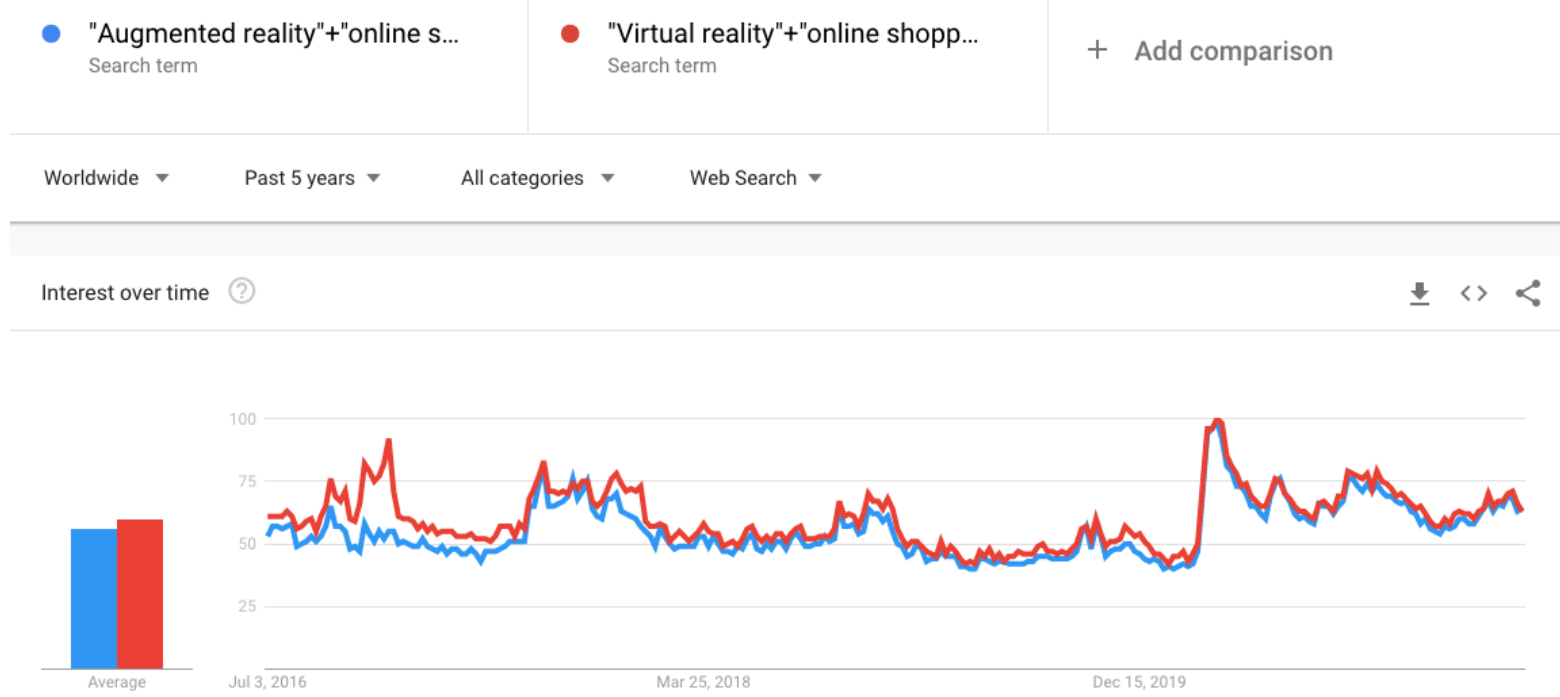

Fig. 1. Google Trends Results for Keyword Searches (as on 29 Jun, 2021)

ISSN: 2582-4252 (online) 
In online retail industry, the use of VR/AR technology and future possibilities are analyzed in different contexts ranging from real estate to clothing and makeup [15]. Product oriented possibilities are often observed in most of the cases. Virtual apparel fitting, haptic gloves and product visualization provide online buyers a visual appeal by assuring them that they are getting precisely what they want. Electric pulses in the hand may be used to feel the texture, weight, and pattern of a product when doing online shopping with haptic gloves. When compared to the traditional online shopping features, this technology will enhance the shopping experience by multiple folds. For instance, LensKart application offers online frame selection based on the suitability of customer's face and Nykaa applications offers the selection of make-up products based on the customer skin tone by using virtual fitting based on AR technology [16]. The customers can get a real-time feedback based on the apparel that looks good on them by using virtual fitting, which enables them to make the right purchase decision. The hardware involved in VR/AR technologies has been investigated by certain researchers, while others analyzed the online store navigation process [17]. Table 1 discusses about the key research areas and research findings in the VR/AR technologies, which are used in retail industries as studied in various literature.

Table 1: VR/AR in Retail Industry

\begin{tabular}{|c|c|c|}
\hline Reference & Key Research Area & Research Findings \\
\hline [18] & Touch simulation & $\begin{array}{l}\text { The texture, weight and pattern of the product may be felt by } \\
\text { means of electric pulses in hand while doing online shopping } \\
\text { with the use of haptic gloves. }\end{array}$ \\
\hline [19] & Apparel fitting & Customers can choose clothes that fit them best. \\
\hline$[20]$ & Personal Navigation & Familiarity in user interface increases usage duration \\
\hline [21] & Data Visualization & $\begin{array}{l}\text { Provides optimized shopping experience with real time data } \\
\text { visualization }\end{array}$ \\
\hline$[22]$ & 3D interaction & $\begin{array}{l}\text { Improves the shopping experience by enabling user to interact } \\
\text { with the virtual environment. }\end{array}$ \\
\hline
\end{tabular}


Journal of Innovative Image Processing (JIIP) (2021)

Vol.03/ No. 02

Pages: 144-156

https://www.irojournals.com/iroiip/

DOI: $\underline{\text { https://doi.org/10.36548/jiip.2021.2.006 }}$

\section{AR and VR research and applications}

Key phrases like retail, shopping, online, virtual and augmented are considered throughout this review. The search frequency of these words are intriguing and unexpected from the observed trends. User experience is commoditized outside the physical or retail items using VR/AR technologies in applications involving tourism, museum and other services. Appropriate design plays a major role in these application [23]. VR shopping experience is leveraged to improve the market share and profits in the fashion industry. The VR/AR based e-commerce platforms are expanding largely requiring great research focus in the design techniques of this technology. The customer buying behavior is largely affected by the user interface (UI) or user experience (UX) factors that is reflected in the underlying theme of the application [24]. The technical and experiential development is significant based on its contribution to the e-commerce VR/AR application and its monetary convergence despite the various topics addressed by the literature. The AR technology is widely used in Instagram filters.

Online shopping customers are attracted towards user-friendly interfaces, simple checkout procedures, clear navigational structures, updated information, efficient search engines and comfortable page layouts as observed in the website design research [25]. The VR shop design may be applied with these design features. As customer navigation functions and new store design conditions operate under diversified concepts, it is essential to regulate the 3D environment in the store layout. The VR/AR literature has certain elusive human computer interface (HCI) theories that are to be addressed to meet the research requirements of the applications [26]. The shopping malls and other commercial premises must be appropriately designed to increase its commercial benefits. Experience is a critical term in this process apart from design. Interaction between the consumer and the brand, environment or product is termed as the user experience. The shopping environment is affected in a holistic manner with regard to the physical, social, affective, emotional and cognitive responses. Instead of design artefacts, much focus is given to designing experiences by various researchers. The commercial art of modern days are dependent on experience design [27]. Here, a staged experience is combined with various demonstrations of a product over a single theme. 
Table 2: Factors Affecting User Shopping Experience

\begin{tabular}{|l|l|l|}
\hline Impact & \multicolumn{1}{|c|}{ Factor } & \multicolumn{1}{|c|}{ Observation } \\
\hline \multirow{3}{*}{ Direct } & Interactivity & $\begin{array}{l}\text { Affects the performance, communication, options and } \\
\text { convenience of the user. }\end{array}$ \\
\cline { 2 - 3 } & Visualization & $\begin{array}{l}3-\text { D model or virtual fitting enables the user a better product } \\
\text { selection idea. }\end{array}$ \\
\cline { 2 - 3 } & Service & $\begin{array}{l}\text { Virtual character with facial expression and body motions } \\
\text { rather than text chat interface improves the user interaction and } \\
\text { service quality }\end{array}$ \\
\hline \multirow{3}{*}{ Indirect } & Accessibility & $\begin{array}{l}\text { Increases accessibility from remote locations and enable 24/7 } \\
\text { usability }\end{array}$ \\
\cline { 2 - 3 } & Technology & $\begin{array}{l}\text { Software and hardware compatibility and timely updations } \\
\text { increases the reliability }\end{array}$ \\
\cline { 2 - 3 } & Aesthetics & $\begin{array}{l}\text { Increases the product demonstration and showcasing } \\
\text { possibilities with increased visual comfort. }\end{array}$ \\
\hline
\end{tabular}

Table 2 compares the direct and indirect factors that are affecting the overall user shopping experience by using VR/AR technologies in the retail industry. The inadequacies of ecommerce have to be met by the VR and AR technologies by comparing the in-store and online shopping environments. Comparison of the product style, size and quality may be a challenge to the consumers due to the lack of product perception, which includes the tactile and physical features excluded in e-commerce [28]. The designers must be updated with the recent market trends, technological innovation, and technology acceptance by the consumer, consumer characteristics and perception in order to design an effective shopping environment. These factors enhance the consumer shopping experience by contributing towards setting up efficient retail and marketing strategies.

Similar to healthcare and education, various possibilities exist in the retail industry with the use of VR/AR technologies. It provides innovative and unique user experiences with fully immersive environment. Complete creation of virtual environment similar to the entertainment domain and utilization in various perspectives of shopping is a major advantage of this technology. However, with the use of a VR headset, the user loses sight of the real environment, leading to possible damage or injuries to the surroundings [29]. This 
issue is reduced to a certain extent with the AR technology as it incorporates the virtual element in the real-world background. In both VR and AR, space limitation is a major challenge. The cost of VR headsets is a potential challenge questioning the affordability and user friendly nature of the device. Continuous use of the device for long time duration may cause motion sickness as the refresh frequency of the image sequence is low. Increasing the refresh frequency of the images may reduce this limitation to a certain extent [24]. Various challenges have been acknowledged by the VR and AR technology developers and users. Privacy protection is not very advanced yet in the VR/AR systems causing data breach and security issues leading to information leakage. Time consumption, technical complexity and cost are the overall major challenges in creating a virtual environment.

\section{Research Gaps with VR/AR}

According to the literature, effective VR/AR based e-commerce environment design offers latest technology interaction opportunities while reconsidering traditional HCI theory, and retail environment interactivity level are some of the prominent factors that contribute to consumers' access to VR or AR technology in recent years. Over the past decade, several academic and industrial researches have been undertaken under this domain [25]. The virtual and augmented e-commerce designs have to address the most prominent questions at a holistic level. Existing research has answered several key questions while ensuring consumer satisfaction. However, the e-commerce shopping is largely influenced by variable concerns, which is a significant deficiency in the existing literature. Brand communication, purchase time frame, prior VR experience, interaction effect, age of the user and other moderating variables may be examined to address this research gap [26-27]. The virtual retailing and the impact of consumer perception based on various independent variables must be considered as a future research direction.

Major gaps are identified in over half of the existing literature in the VR and AR e-commerce design domain. The online environment social stimuli are the main elements of the framework. A social dimension must be met to develop the system with appropriate human computer interaction environment [28]. In VR shopping, eye-tracking is a prominent 
Journal of Innovative Image Processing (JIIP) (2021)

Vol.03/ No. 02

Pages: $144-156$

https://www.irojournals.com/iroiip/

DOI: https://doi.org/10.36548/jiip.2021.2.006

parameter. The academic literature often fail to address the mobile eye-tracking technology assessment, efforts and challenges. The VR studies need to focus on the incorporation schemes of eye-tracking techniques in future work. It would be an interesting aspect to engage with the disadvantages of shoppers in terms of design and user interface which is missing in most literature studies [29]. The relationship between sales and customer behavior in studies related to VR/AR are also not covered in most studies. Within multiple sales channels, the motivation and behavior of the consumer must be understood while the interactive technologies and consumer responses must be expanded by investigating the human computer interaction aspects in a more detailed manner. The acceptance of these technologies may also be analyzed to bridge these research gaps.

When compared, the results from real-world environment and those obtained from a laboratory environment, vary by a factor of $8 \%$ by creating a large impact on the research findings. High external validity for theoretical development is essential. For this purpose, rather than laboratory experiments, field tests must be run by manipulating certain store variables with permission from VR retailers in future research work. The dependent variables and their pre and post utilization measurements of the VR experience and its impact on the retail profit must be examined for further investigation [30]. Experience of participants with VR and acceptance of the immersive environment and technology can be examined based on the financial outcomes of the retail industry as observed from longitudinal study.

\section{Conclusion}

The interdisciplinary nature of VR and AR has led to various academic research domains by including management, marketing and technology contributing towards the fragmented nature of the research. A future research agenda is formulated for retail applications using VR and AR technologies by analyzing the chronological developments and critical review of the pros, cons and research gaps. The best potential of the VR and AR technology in the context of retail industry may be realized using certain possible directions. In online retailing, the successful implementation and adoption of VR and AR technology interfaces enhances the shopping experience and makes it more consumer friendly. While designing an efficient 
virtual shopping environment, human-computer interaction, retailing, marketing and various other disciplines must be analyzed along with the cooperation and shared understanding between them. Another significant factor emerging in this domain is collaboration between retailers and the VR/AR technology providers. Consumer characteristics in terms of technology acceptance, market trends, need for innovation, implementation requirements, dealing with barriers and so on must be understood by the technology provider as well as the retailer. Sharing insights and comparing views enhances and enriches the customer shopping experience while implementing effective retailing and marketing strategies by combining the skills and forces. According to certain critics, rather than becoming a viable in-store solution, the consumer attention is gained in large through VR technology. With minimum returns, it is considered that the implementation cost and time for this technology is high leading very few retailers to adopt this technology.

For experience driven minor retailers, this technology will be a special add-on feature. A complete understanding must be achieved regarding the evolution of this phenomenon in combination with various disciplines in future research. In 2016, Mark Zuckerberg, founder of Facebook predicted that the VR headsets and a normal pair of glasses will look alike leading to a higher level of acceptance of consumers with advanced technologies. The shoppers are attracted in a potential manner with fashionably acceptable, natural, useful, easy to interact, subtle, discrete and socially acceptable features offered by this technology. The VR consumer adoption is driven strongly with the help of mobile technology in the current scenario. As this technology is comfortable and familiar to the users, the barriers in adoption of new technology is reduced with the utilization of mobile devices for interaction with the retail products providing a VR experience without the need to purchase a VR headset.

Various challenges have been acknowledged by the VR and AR technology developers and users. Privacy protection is not very advanced yet in the VR/AR systems causing data breach and security issues leading to information leakage. For users with mobile devices who do not need an extra viewing device, this challenge may not be significant. However, when data has to be transmitted between devices, the security and privacy of the information must be taken into consideration. In online retailing, there is a continuous advancement and evolution of VR and AR technologies. From research insights, the managerial implications, price accessibility, 
Journal of Innovative Image Processing (JIIP) (2021)

Vol.03/ No. 02

Pages: 144-156

https://www.irojournals.com/iroiip/

DOI: $\underline{\text { https://doi.org/10.36548/jiip.2021.2.006 }}$

acceptability, privacy concerns and other perceived barriers must be examined to increase the utilization and acceptance of the technology at the consumer end. Various contributions are offered by this review in an overall perspective. In the retail context, the use of VR and AR applications and their chronological developments are reviewed in a critical and detailed manner. The significance of various subjects across different domains and their importance is synthesized. The research agenda is highlighted to make way for future research inquiry framework that acts as a catalyst to future research arenas.

\section{References}

[1] Caboni, F., \& Hagberg, J. (2019). Augmented reality in retailing: a review of features, applications and value. International Journal of Retail \& Distribution Management.

[2] Singh, Devendra, Pawan Kumar Pant, Himanshu Pant, and Dinesh C. Dobhal. "Robust Automated Machine Learning (AutoML) System for Early Stage Hepatic Disease Detection." In Intelligent Data Communication Technologies and Internet of Things: Proceedings of ICICI 2020, pp. 65-76. Springer Singapore, 2021.

[3] Shakya, Subarna, and S. Smys. "Reliable Automated Software Testing Through Hybrid Optimization Algorithm." Journal of Ubiquitous Computing and Communication Technologies (UCCT) 2, no. 03 (2020): 126-135.

[4] Parekh, P., Patel, S., Patel, N., \& Shah, M. (2020). Systematic review and metaanalysis of augmented reality in medicine, retail, and games. Visual computing for industry, biomedicine, and art, 3(1), 1-20.

[5] Ponmaniraj, S., Tapas Kumar, and Amit Kumar Goel. "Intrusion Detection: Spider Content Analysis to Identify Image-Based Bogus URL Navigation." In Intelligent Data Communication Technologies and Internet of Things: Proceedings of ICICI 2020, pp. 603-617. Springer Singapore, 2021.

[6] Cruz, E., Orts-Escolano, S., Gomez-Donoso, F., Rizo, C., Rangel, J. C., Mora, H., \& Cazorla, M. (2019). An augmented reality application for improving shopping experience in large retail stores. Virtual Reality, 23(3), 281-291.

[7] Smys, S. "A Survey on Internet of Things (IoT) based Smart Systems." Journal of ISMAC 2, no. 04 (2020): 181-189. 
Journal of Innovative Image Processing (JIIP) (2021)

Vol.03/ No. 02

Pages: $144-156$

https://www.irojournals.com/iroiip/

DOI: $\underline{\text { https://doi.org/10.36548/jiip.2021.2.006 }}$

[8] van Esch, P., Arli, D., Gheshlaghi, M. H., Andonopoulos, V., von der Heidt, T., \& Northey, G. (2019). Anthropomorphism and augmented reality in the retail environment. Journal of Retailing and Consumer Services, 49, 35-42.

[9] Vivek Sidhaarthan, S., Anand Mukul, P. Ragul, R. Gokul Krishna, and D. Bharathi. "Offline 3D Indoor Navigation Using RSSI." In Intelligent Data Communication Technologies and Internet of Things: Proceedings of ICICI 2020, pp. 831-846. Springer Singapore, 2021.

[10] Perannagari, K. T., \& Chakrabarti, S. (2019). Factors influencing acceptance of augmented reality in retail: insights from thematic analysis. International Journal of Retail \& Distribution Management.

[11] Vijayakumar, T., Mr R. Vinothkanna, and M. Duraipandian. "Fusion based Feature Extraction Analysis of ECG Signal Interpretation-A Systematic Approach." Journal of Artificial Intelligence 3, no. 01 (2021): 1-16.

[12] Fan, X., Chai, Z., Deng, N., \& Dong, X. (2020). Adoption of augmented reality in online retailing and consumers' product attitude: A cognitive perspective. Journal of Retailing and Consumer Services, 53, 101986.

[13] Sahila, K. M., and Bibu Thomas. "Secure Digital Image Watermarking by Using SVD and AES." In Intelligent Data Communication Technologies and Internet of Things: Proceedings of ICICI 2020, pp. 805-818. Springer Singapore, 2021.

[14] Nikhashemi, S. R., Knight, H. H., Nusair, K., \& Liat, C. B. (2021). Augmented reality in smart retailing: A (n)(A) Symmetric Approach to continuous intention to use retail brands' mobile AR apps. Journal of Retailing and Consumer Services, 60, 102464.

[15] Chen, Joy Iong Zong, and P. Hengjinda. "Early Prediction of Coronary Artery Disease (CAD) by Machine Learning Method-A Comparative Study." Journal of Artificial Intelligence 3, no. 01 (2021): 17-33.

[16] Jung, T., \& tom Dieck, M. C. (2018). Augmented Reality and Virtual Reality. Empowering Human, Place and Business. Cham: Springer International Publishing.

[17] Yadav, Nikhil, Omkar Kudale, Aditi Rao, Srishti Gupta, and Ajitkumar Shitole. "Twitter Sentiment Analysis Using Supervised Machine Learning." In Intelligent Data Communication Technologies and Internet of Things: Proceedings of ICICI 2020, pp. 631-642. Springer Singapore, 2021. 
Journal of Innovative Image Processing (JIIP) (2021)

Vol.03/ No. 02

Pages: 144-156

https://www.irojournals.com/iroiip/

DOI: $\underline{\text { https://doi.org/10.36548/jiip.2021.2.006 }}$

[18] Bonetti, F., Pantano, E., Warnaby, G., Quinn, L., \& Perry, P. (2019). Augmented reality in real stores: empirical evidence from consumers' interaction with AR in a retail format. In Augmented Reality and Virtual Reality (pp. 3-16). Springer, Cham.

[19] Adam, Edriss Eisa Babikir. "Evaluation of Fingerprint Liveness Detection by Machine Learning Approach-A Systematic View." Journal of ISMAC 3, no. 01 (2021): 16-30.

[20] Zhang, J. (2020). A Systematic Review of the Use of Augmented Reality (AR) and Virtual Reality (VR) in Online Retailing (Doctoral dissertation, Auckland University of Technology).

[21] Hariharakrishnan, Jayaram, and N. Bhalaji. "Adaptability Analysis of 6LoWPAN and RPL for Healthcare applications of Internet-of-Things." Journal of ISMAC 3, no. 02 (2021): 69-81.

[22] Xue, L., Parker, C. J., \& McCormick, H. (2019). A virtual reality and retailing literature review: Current focus, underlying themes and future directions. Augmented Reality and Virtual Reality, 27-41.

[23] Suma, V., and Wang Haoxiang. "Optimal Key Handover Management for Enhancing Security in Mobile Network." Journal of trends in Computer Science and Smart technology (TCSST) 2, no. 04 (2020): 181-187.

[24] Jung, T. (2019). Augmented Reality and Virtual Reality. The Power of AR and VR for Business. Springer Nature Switzerland AG..

[25] Joe, Mr C. Vijesh, and Jennifer S. Raj. "Location-based Orientation Context Dependent Recommender System for Users." Journal of trends in Computer Science and Smart technology (TCSST) 3, no. 01 (2021): 14-23.

[26] Chandan, T. (2021). EXPLORATION OF AUGMENTED REALITY AND VIRTUAL REALITY IN THE RETAIL INDUSTRY. International Journal of Modern Agriculture, 10(2), 431-442.

[27] Adam, Edriss Eisa Babikir. "Deep Learning based NLP Techniques In Text to Speech Synthesis for Communication Recognition." Journal of Soft Computing Paradigm (JSCP) 2, no. 04 (2020): 209-215.

[28] Upadhyay, G. K., Aggarwal, D., Bansal, A., \& Bhola, G. (2020, February). Augmented Reality and Machine Learning based Product Identification in Retail 
Journal of Innovative Image Processing (JIIP) (2021)

Vol.03/ No. 02

Pages: $144-156$

https://www.irojournals.com/iroiip/

DOI: https://doi.org/10.36548/jiip.2021.2.006

using Vuforia and MobileNets. In 2020 International Conference on Inventive Computation Technologies (ICICT) (pp. 479-485). IEEE.

[29] Haoxiang, Wang, and S. Smys. "Overview of Configuring Adaptive Activation Functions for Deep Neural Networks-A Comparative Study." Journal of Ubiquitous Computing and Communication Technologies (UCCT) 3, no. 01 (2021): $10-22$.

[30] Teh, C., Phang, C. W., Chong, A. Y. L., \& Guo, Z. (2021, January). Augmented Reality in Offline Retail: Integrating the Affordance and Means-End Chain Perspectives. In Proceedings of the 54th Hawaii International Conference on System Sciences (p. 1613).

\section{Author's Biography}

T. Senthil Kumar, is currently working as an Associate Professor, in the Computer Science and Engineering Department, at Amrita School of Engineering, Amrita Vishwa Vidyapeetham, TamilNadu, India. His major areas of interest are Real time Image Processing, Computer Vision and Pattern Recognition, Image Processing Systems and algorithms for Medical, Industrial, Embedded and Vision assisted Intelligent Robotic Systems, video communication, biomedical imaging, electronic imaging, image and video systems, and remote sensing.

ISSN: 2582-4252 (online) 